\title{
Correlation Coefficient Control For Subgrade Settlement Curve Fitting
}

\author{
Ruijun Song ${ }^{a}$, ZhangJun Dai ${ }^{b}$, Shanxiong Chen, Jian Li, Zecheng Chi \\ State Key Laboratory of Geomechanics and Geotechnical Engineering, Institute of Rock and Soil \\ Mechanics, Chinese Academy of Sciences, Wuhan 430071, China \\ a398449801@qq.com, b527353357@qq.com
}

\begin{abstract}
Keywords: regression curve; correlation coefficient; small scale, large fluctuations; settlement; reliability.

Abstract. The measured settlement curves of high-speed railway are presented with the characteristic of "small scale, large fluctuations", which is difficult to reach the requirements of curve regression coefficient with 0.92 on specification. This was done by adopting the method of mathematical simulation and hyperbola fitting to study the change rules of correlation coefficient on subgrade simulated settlement curve, under different conditions. From the analysis result, smaller value of parameter $a$ and $b$, will have great influence on curve correlation coefficient, otherwise, the parameter $c$ becomes the major factor; When $c$ value is greater than $1.5 \mathrm{~mm}$, the parameter $b$ becomes the major factor; Considering the premise, it is recommended that in order to observe the total settlement amount for working point $<10(5) \mathrm{mm}$ that can determine the post-Settlement deformation trend condition, the correlation coefficient of the regression curve minimum standards can be relaxed from 0.92 to 0.8 .
\end{abstract}

\section{Introduction}

In order to realize the requirements of fast, comfortable, safe, the high-speed railway for passenger-dedicated lines should be strictly control the post-construction settlement $[1,2]$. Because of the smaller measured settlement of passenger dedicated line, especially settlement increment and measurement system error are in the same order of magnitude after the completion of the main construction. The situations that observations appear leap (including the falling and rising) or continuous several settlement observation data trends in contrast to the conventional (settlement acceleration or uplift) are more, and the measured settlement curves are more presented with the characteristics of "small scale, large fluctuations", which is difficult to reach the requirements of curve regression coefficient with 0.92 according to the specification of "Technical Guidelines for assessing ballastless track laying conditions"(2006).

\section{The characteristics of settlement observations in high speed railway}

According to the requirements of "Technical Guidelines for assessing ballastless track laying conditions"(2006), the post construction settlement of the whole line should be less than $15 \mathrm{~mm}$ with the limit of ballastless track [3]. But from the settlement deformation data of the Hubei Intercity Railroad, except for the subgrade settlement deformation which is located in the part of soft soil roadbed preloading areas can reach $40 \sim 50 \mathrm{~mm}$, most of the subgrade settlement deformation is less than $15 \mathrm{~mm}$, and bridges and tunnels are less than $5 \mathrm{~mm}$. therefore, the project of the measured settlement deformation is small in Hubei Intercity Railroad, and the settlement deformation increment will be smaller after stop loading, which will lead to observations appear larger fluctuation caused by the system error.

The influence characteristic of the noise to the correlation coefficient of subgrade settlement curve fitting

The simulated settlement curve. The subgrade measured settlement curve of the high speed railway inevitably exist noise, in older to investigate the influence characteristic of the noise to the correlation coefficient of subgrade settlement curve fitting, adopting mathematical simulation method to study the 
change rules of correlation coefficient on subgrade simulated settlement curve under different conditions.

Engineering practice shows that the development of foundation settlement with time approximately uses hyperbolic to describe [4]. Therefore, selecting a set of ideal settlement curve satisfied formula (3.1) without noise.

$$
\begin{aligned}
& S_{t}=S_{0}+\frac{t}{a+b t} \\
& S_{f}=S_{0}+\frac{1}{b}
\end{aligned}
$$

In the formulas, $S_{t}$ - the settlement of the moment $t, S_{f}-$ the final settlement $(t=\infty), S_{0}-$ the initial settlement $(t=0), a 、 b$-obtaining the coefficient of linear regression from measurement data with force remained unchanged.

The actual settlement curve of the project has a certain fluctuation, in order to simulate the settlement curve with noise, adding a random parameter behind the ideal hyperbolic equation $\mathrm{r}(\mathrm{x})$, and the formula(3.3)as two simulated settlement curves of the experiment.

$$
S=\frac{t}{a+b t}+\operatorname{cr}(x)
$$

In the formula, $\mathrm{S}$ - simulating the measured settlement curve, $\mathrm{c}$ - parameter (the volatility to control the random function). By setting the variation range of random function in excel table can obtain the random function.

The simulated settlement curve is determined by the parameters a, b, c. From the equation, a value is controlling the convergence speed of the simulated settlement curve, $b$ value is controlling the cumulative settlement of the simulated settlement curve, $\mathrm{c}$ value is controlling the fluctuation range of the simulated settlement curve.

Simulation Scheme. In order to study the change rules of correlation coefficient on subgrade simulated settlement curve under different conditions, by adjusting the coefficients $\mathrm{a}, \mathrm{b}$, c values to simulate the subgrade settlement curve in different conditions, and then using the hyperbola to fit and analyze the simulation curve.

i.e $a=4, b=0.1, c=1$ (if $b=0.1, \mathrm{~S}_{\mathrm{f}}=10 \mathrm{~mm}$ and if $c=1$, the variation range is between $\pm 1 \mathrm{~mm}$ ), which arrived at the correlation coefficient $\mathrm{R}=0.96$ with hyperbolic fitting method. On the basis of this two fixed coefficient discussed the influence of the correlated coefficient from each values.

According to the samples, when $a$ value is divided into 40、27、4、1.4、1、0.4; $b$ value is divided into $0.2 、 0.1 、 0.05 、 0.033 、 0.017 、 0.01 ; c$ value is divided into $0.5 、 1 、 1.5 、 2 、 3$, the simulation curve assumes that the observation period is one year after finishing the main part of the project.

The impact for correlation coefficient (R) from $\boldsymbol{a} 、 \boldsymbol{b} 、 \boldsymbol{c}$ values. Adjusting the $a, b 、 c$ values separately, and then using the hyperbola to fit and analyze the simulation curve. Simulation curve and regression curve are both drawn in the figure 3.1, and the correlation coefficient changed with $a, b, c$ values are listed in table 3.1. 
Table 3.1 The corresponding correlation coefficient of varied $a, b 、 c$ values

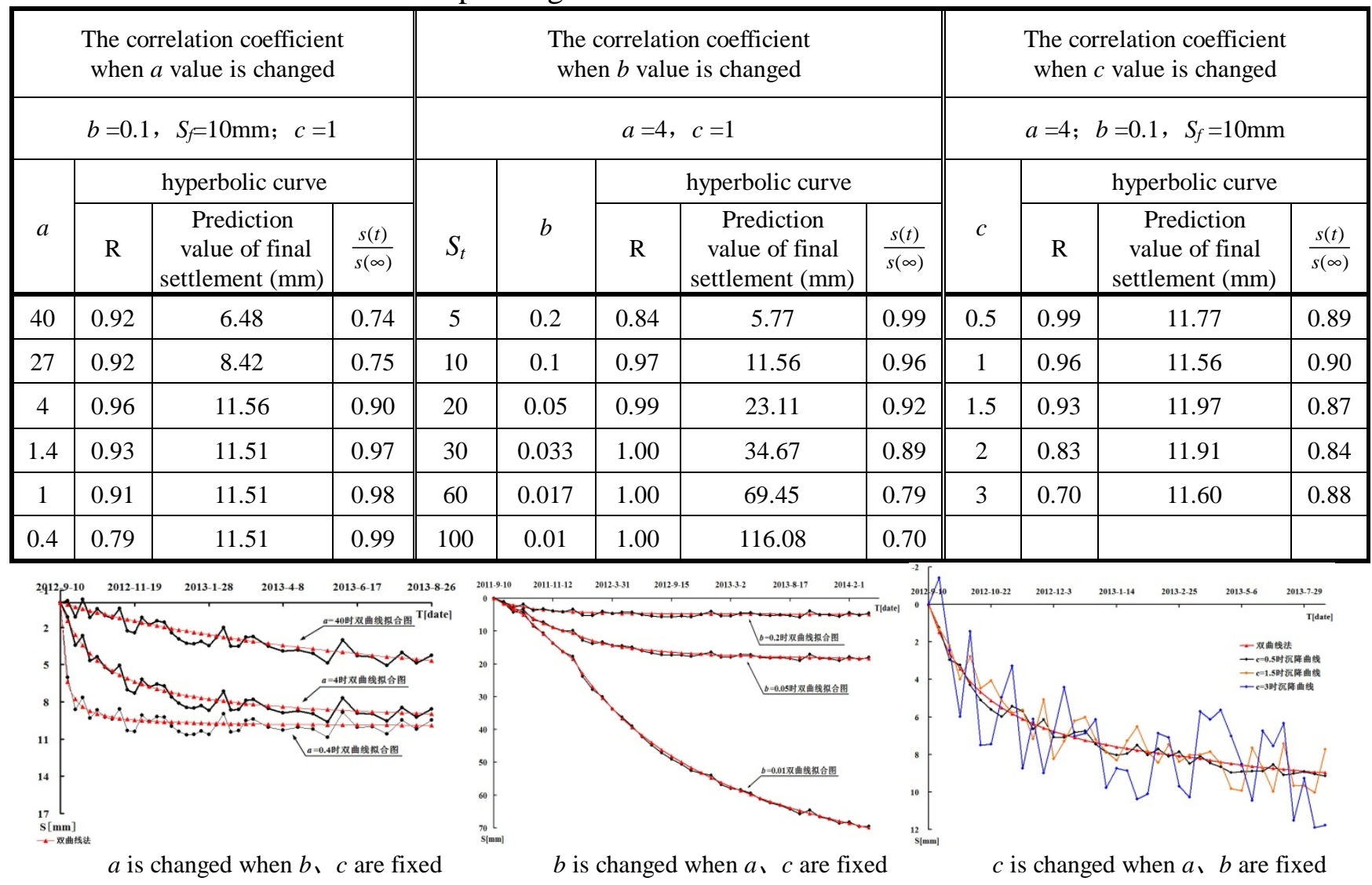

Fig. 3.1 The comparison diagram of hyperbolic fitting prediction and settlement curve when $a, b 、 c$

values are different

1) The impact for correlation coefficient (R) from a value. From fig. 3.1, when $b 、 c$ values are fixed and $a$ value is bigger, the settlement is slow-growth along with the time. When $a$ value is smaller, the settlement starts steep and slow down gradually in the mid to late and later flattens; Table 3.1 shows that when $a$ value is changed from 40 to 4 , the correlation coefficient of curve regression is changed from 0.92 to 0.96 . As $a$ value is further reduced, the correlation coefficient is reduced gradually.

In terms of engineering, the observed settlement usually is rapid development in early stage and later flattened over time, therefore, $a$ value tends not to be too large. When $a$ value is smaller, the settlement curve converges quickly with the higher rate at the beginning and reaches stable value in a short time. At the moment, the regression coefficient of the settlement curve is smaller on the whole, which is the result of the significant fluctuations in the data caused by the rapid development of the settlement in the early stage. But from the whole change trend, the settlement is basically stable in the mid to late, the correlations of fitted curves and measured curves are higher in this section, which is a reliable prediction overall.

2) The impact for correlation coefficient (R) from $b$ value. From fig 3.1 and table 3.1 , there is a better forecast with the method of hyperbola when $b$ value is smaller. When $b$ value is less than 0.125 , the correlation coefficient of curve is not smaller than 0.92 which is requirement of the specification. When $b$ value is too small, correlation coefficient can still reach 0.92, But at the moment, the fitted hyperbolic does not meet the requirements of the specification for stability. When $b$ value is bigger, the measured deformation of sedimentation is small. Although the overall regression coefficient of the curve is smaller and it cannot meet the requirements of specifications at the moment, it is the most result of the significant fluctuations in the data caused by the measurement system error, especially settlement increment and measurement system error are in the same order of magnitude after the completion of the main construction, the data appeared larger fluctuations will greatly influence the correlation coefficient of regression curve. Therefore, it should consider eliminating the error effectively through data processing and guarantee the reliability of settlement analysis data. 
3) The impact for correlation coefficient (R) from c value. From fig 3.1 and table 3.1, the smaller $c$ value is the higher correlation coefficient of hyperbolic method to predict. As for the existing observation techniques in practice, the improvement of monitoring accuracy is limited, meanwhile various interferences of the engineering on physical conditions is so hard to avoid, so the measured settlement curves are more presented with the characteristics of "small scale, large fluctuations" which results in a bigger $c$ value and hard to reach the 0.92 of the correlation coefficient. But from the whole change trend, the settlement is basically stable, and other evaluation indexes can meet the requirements of the "Technical Guidelines for assessing ballastless track laying conditions"(2006). Hence, it can determine the settlement is able to meet the conditions for unballasted track laying.

\section{Conclusion}

The followings are the impacts of prediction with hyperbola to correlation coefficient:

The smaller $a$ value will have a great influence on correlation coefficient, When $b$ value is smaller, the fluctuation of curve has greater effects on correlation coefficient, but beyond that, the parameter $c$ is the major factor; When controlling the fluctuation range of settlement curve on $c$ value is less than 1.5 , the prediction can be achieved. Beyond this range, the cumulative settlement of actual settlement curve will have bigger influence on the correlation coefficient, and at present, the parameter $b$ is the major factor.

The correlation coefficient is a statistic index which is to illustrate the close degree of the correlativity between the two phenomena under the condition of linear correlation. In mathematics, the standard for judging two variables on the close degree of linear correlation as the followings: When $0.5 \leq \mathrm{R}<0.8$, it's called significant correlation and when $0.8 \leq \mathrm{R}<1$, it's called high correlation. Therefore, the reliability of the prediction will not be significantly reduced when the standard on correlation coefficient of the regression curve relaxes to 0.85 . Because when $R \geq 0.85$, the curve of regression and the measured are high correlation, which is reliable to predict.

Because of the smaller measured settlement of passenger dedicated line, measurement system error of the observations will cause larger fluctuation relative to the smaller settlement and the correlation coefficient of the regression curve is hard to reach 0.92. But from the whole change trend, the settlement is basically stable, and other evaluation indexes can meet the requirements of the "Technical Guidelines for assessing ballastless track laying conditions"(2006). Hence, it can determine the settlement is able to meet the conditions for unballasted track laying.

Consequently, when the total settlement is too small, the measurement system error on the correlation coefficient of regression curve has a great influence. Considering the premise, it is recommended that in order to observe the total settlement amount for working point $<10(5) \mathrm{mm}$ that can determine the post-Settlement deformation trend condition, the correlation coefficient of the regression curve minimum standards can be relaxed from 0.92 to 0.8 .

\section{References}

[1] Wang Xingyun. Research of Prediction Method for Subgrade Settlement on Railway Passenger Dedicated Line [D]. Wu Han, Dissertation for the Master of Geotechnical Engineering, 2009.

[2] Liu Fang, Pan Weidong, Wang Guangyong. Study on Settlement Behavior and Deformation Predict of Subgrade for Wu Guang Passenger Line [J]. Jangsu Constructions, 2008, (05): 0061-02.

[3] Chen Hongyun, Ma Jianlin, Qin Xiaogang, Hu Weiming. Research on Hyperbolic Methods Fitting Settle-ment of the Subgrade in High Speed Railway [J]. Journal of Safety and Environment, 2014, (04): 0011-04. 
[4] Wang Xingyun, Chen Shanxiong, Yu Fei, Zhou Bo. Study of Applicability of Curve Fitting Methods in small Settlement of Subgrade [J]. Rock and Soil Mechanics, 2009, (09): 2763-07. 